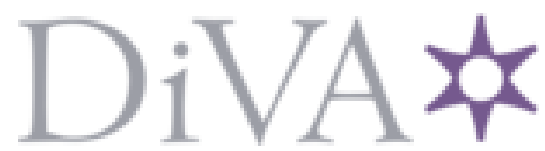

http://www.diva-portal.org

This is the published version of a paper presented at IEEE/OSA Asia Communication and Photonics Conference.

Citation for the original published paper:

Li, J. (2017)

Dynamic bandwidth slicing for service migration in passive optical network based mobile backhaul

In:

N.B. When citing this work, cite the original published paper.

Permanent link to this version:

http://urn.kb.se/resolve?urn=urn:nbn:se:kth:diva-256510 


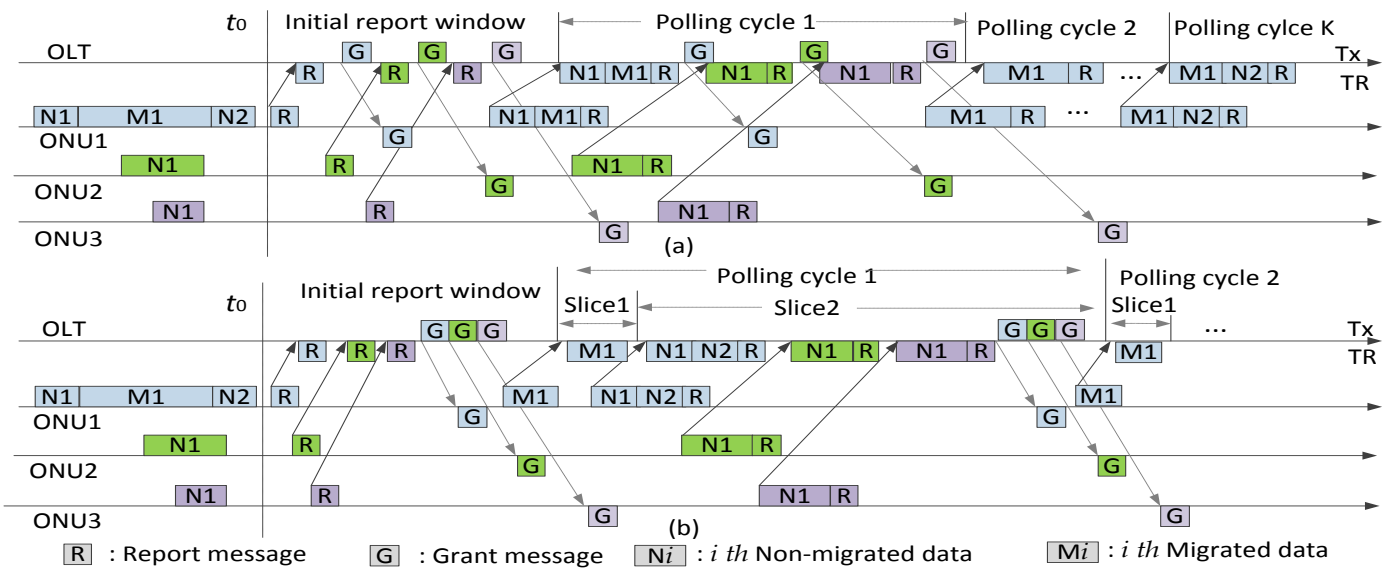

Fig. 1: (a) The traditional dynamic bandwidth allocation algorithm and (b) the proposed dynamic bandwidth slicing algorithm.

arrives, which requires multiple polling cycles to be transmitted. As a consequence, non-migrated data $N 2$ at $\mathrm{ONU}_{1}$ that arrive after $M 1$, have to wait and experience high latency. In the proposed DBS algorithm (see Fig. 1(b)), the cycle time can be dynamically divided into several slices, which are provisioned to different kind of traffic. The proposed DBS algorithm is also based on the report and grant mechanism. If there is no migrated data to be transmitted in the polling cycle, the proposed DBS algorithm performs in the same way as the classical DBA algorithm. Once service migration occurs, the resource controller located at the OLT calculates the lengths of the slices for both migrated and nonmigrated traffic according to the information included in the report messages, namely the size and deadline for the migrated data. The granted time slots for Slice 1 (SL1) are allocated to the migrated data according to the ascending order of the deadline for finishing migration. In Slice $2(S L 2)$ the time slots are granted for the non-migrated traffic and are proportional to the requested time slots. Note that the same principle as the proposed DBS can also be used in the downstream. The pseudocode of the proposed DBS is presented in Fig. 2, while the corresponding parameters are explained in Table 1 .

\section{Performance evaluation}

The proposed DBS algorithm is evaluated by numerical simulations with MATLAB in terms of average migration delay of migrated data as well as average packet latency and jitter of non- migrated traffic. We consider a TWDM-PON having the time slots for each wavelength scheduled separately, i.e., separate time and wavelength scheduling [8]. The performance of the proposed DBS is tested based on EPON protocol. The interleaved polling with adaptive cycle time (IPACT) is used as the benchmark [9]. The number of ONUs for one PON is 8, while the round-trip time is 200 us corresponding to the fiber reach of $20 \mathrm{~km}$. The packet processing time at OLT is $0.2 \mathrm{~ms}$ and the maximum polling cycle

Table 1: List of Symbols.

\begin{tabular}{|c|l|}
\hline Symbol & \multicolumn{1}{|c|}{ Descriptions } \\
\hline$R N_{i}$ & $\begin{array}{l}\text { Requested time slot for non-migrated data at the } \\
i^{\text {th }} \text { ONU }\end{array}$ \\
\hline$R_{i, j}$ & $\begin{array}{l}\text { Requested time slot for the migrated data } \\
\text { generated by the } j^{t h} \text { service migration at the } \\
i^{\text {th }} \text { ONU }\end{array}$ \\
\hline$D_{i, j}$ & $\begin{array}{l}\text { Deadline for completely migrating the data } \\
\text { generated by the } j^{t h} \text { service migration at the } \\
i^{\text {th }} \text { ONU }\end{array}$ \\
\hline$R F_{i, j}$ & $\begin{array}{l}\text { Granted time slot for the migrated data generated } \\
\text { by the } j^{t h} \text { service migration from the } i^{\text {th }} \text { ONU }\end{array}$ \\
\hline$C_{m a x}$ & Maximum length of polling cycle time \\
\hline$N$ & Number of the ONUs in one PON \\
\hline$K_{i}$ & Number of the service migrations at the $i^{t h}$ ONU \\
\hline$B R$ & $\begin{array}{l}\text { Transmission time for sending each report and } \\
\text { grant massage }\end{array}$ \\
\hline$B G$ & Guard time between two time slots \\
\hline
\end{tabular}

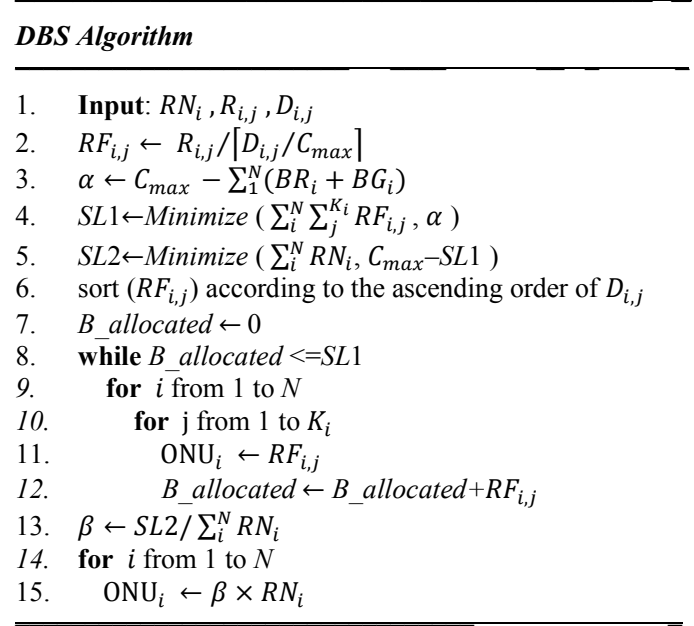

Fig. 2: The pseudocode of the proposed dynamic bandwidth slicing algorithm. 

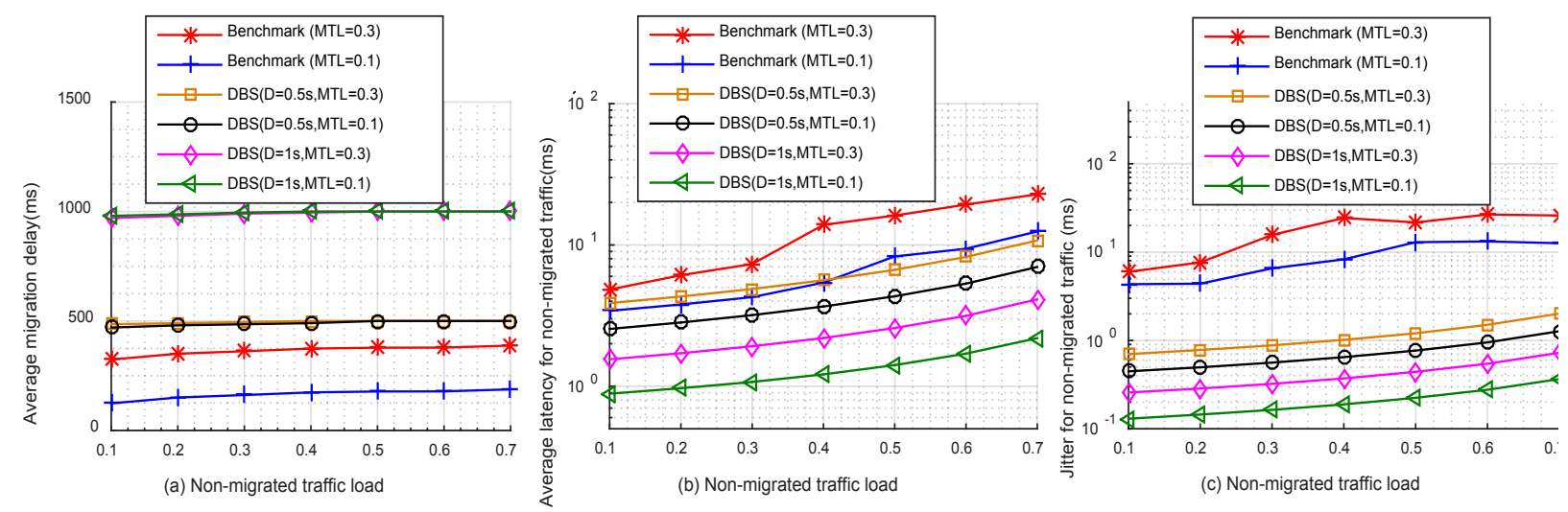

Fig. 3: (a) Average migration delay of the migrated data, (b) average latency and (c) jitter of non-migrated data.

is $2 \mathrm{~ms}[7,9]$. The size of report and grant messages is 64 bytes, while the guard time is 1 us. The line rate is $10 \mathrm{Gbit} / \mathrm{s}$ for both the upstream and downstream. The non-migrated traffic arrives according to Poisson process, and its packet size is uniformly distributed between 64 to 1518 bytes. The Poisson arrivals of service migration is also assumed, and the size of migrated data generated by one service migration is uniformly distributed between 10 and 100 Mbits, which can be fragmented by several 1518-byte Ethernet frames [6,9]. We assume that the buffer size in each ONU is 50 Mbytes, and the simulation time is 100 seconds. Two cases with different migrated traffic load (MTL), i.e., MTL $=0.1$ and 0.3 , are tested. For the proposed DBS algorithm, the impact of different deadlines (D) for the migrated data is also investigated. Fig. 3(a) shows the average migration delay as a function of the non-migrated traffic load for both the benchmark and DBS. In the benchmark, the average migration delay slightly increases with the non-migration traffic load (NTL). Due to FCFS, once the migrated data arrives, it is served immediately regardless of the requirements on deadline. Therefore, the migration delay is pretty low. In the DBS, the average migration delay is $500 \mathrm{~ms}$ when D is set to $0.5 \mathrm{~s}$, while it is $1000 \mathrm{~ms}$ for $\mathrm{D}=1 \mathrm{~s}$, regardless of the MTL. It implies that the required deadline for migration data can be satisfied. Fig. 3(b) shows the average packet delay of the non-migrated traffic. The higher the MTL is, the larger the average packet delay is. However, compared with the benchmark, the average packet latency for the DBS is significantly lower $(30 \%$ for $\mathrm{MTL}=0.1$ and $50 \%$ for $\mathrm{MTL}=0.3$ when $\mathrm{D}=0.5 \mathrm{~s})$. In contrast to the latency, the jitter improvement of the non-migrated data is more obvious. As shown in Fig. 3(c), a reduction by a factor of 10 can be achieved by using the proposed DBS algorithm. Furthermore, when D becomes larger, the packet latency and jitter of the non-migrated data in the DBS can be further decreased. For instance, the average packet latency decreases up to $60 \%$, when D increases from $0.5 \mathrm{~s}$ to $1 \mathrm{~s}$.

\section{Conclusions}

We proposed a dynamic bandwidth slicing algorithm, in which the data generated by service migration in the FeRANs is properly handled. Simulation results reveal that the packet latency and jitter of the non-migrated data can be significantly reduced by partitioning large-size migrated data into smaller pieces while its migration delay constraint is still satisfied. Moreover, with a more relaxed migration delay constraint, the impact on non-migrated data can be further reduced when the proposed DBS algorithm is employed.

\section{Acknowledgements}

This work was supported by the Göran Gustafssons Stiftelse, Natural Science Foundation of Guangdong Province (Grant No. 508206351021) and National Natural Science Foundation of China (Grant No. 61550110240, 61671212).

\section{References}

[1] M. Chiang et al, "Fog and IoT: an overview of research opportunities," IEEE Internet of Things Journal, vol. 3, no. 6, pp. 854-864(2016).

[2] J. Li et al., "Resource management in fog enhanced radio access network to support real-time vehicular services," Proc. ICFEC, Madrid (2017).

[3] M. Jia et al., "Cloudlet load balancing in wireless metropolitan area networks," Proc. INFOCOM, San Francisco (2016).

[4] A. Ashok et al., "Enabling vehicular application using cloud services through adaptive computation offloading,"Proc. VNC, Columbus (2016).

[5] "5G automotive vision" 5G-PPP white paper (2015).

[6] X. Sun et al., “Adaptive avatar handoff in the cloudlet network," IEEE Transaction Cloud computing, vol. PP, no. 99(2016).

[7] J. Li et al., "Optical transport network architecture enabling ultra-low latency for communications among base stations," Proc. OFC Los Angeles (2017).

[8] A. Dixit et al., "Energy efficiency DBA algorithms for TWDM-PONs," Proc. ICTON, Budapest (2015).

[9] G. Kramer et al., "IPACT: A Dynamic Protocol for an Ethernet PON (EPON)” IEEE Com. Mag., vol. 40, no. 2, pp. 74-80 (2002). 